\title{
Statins Counter the Effects of Hyperlipidemia on iNKT Cells
}

\author{
Editorial to: "Statin-Induced Immunomodulation Alters Peripheral Invariant Natural \\ Killer T-Cell Prevalence in Hyperlipidemic Patients" by E. Nakou et al.
}

\author{
Joel Linden
}

Published online: 29 April 2012

(C) Springer Science+Business Media, LLC 2012

Statins are the most widely used lipid-lowering drugs. By inhibiting 3-hydroxy-3-methylglutaryl coenzyme A (HMG$\mathrm{CoA}$ ) reductase they block the rate-limiting step of cholesterol synthesis, resulting in up-regulation of low density lipoprotein (LDL) receptors on liver cell membranes, reduced plasma LDL, and reduced atherosclerosis. In addition to their lipid lowering effects, statins reduce inflammation by effects on many other cells. These include lymphocyte-suppressing effects in patients with elevated cholesterol levels [1]. The anti-inflammatory effects of statins are secondary to HMGCoA inhibition and result from diminished post-translational protein prenylation of small $\mathrm{G}$ proteins such as Ras and Rho, which are important signaling molecules that regulate antigen presenting cells (APCs) and lymphocytes [2]. RhoH has recently been shown to serve as an adaptor molecule that regulates the subcellular localization of the proximal $\mathrm{T}$ cell signaling molecules, lymphocyte-specific protein tyrosine kinase (Lck) and Zeta-chain-associated protein kinase 70 (Zap-70) [3] (Fig. 1).

The antigens that activate most $\mathrm{T}$ cell receptors are peptides presented by MHC on APCs. In a minor subset of lymphocytes known as iNKTcells, certain lipid antigens are presented by the MHC-like molecule, CD1d. The TCRs of iNKT cells are not variable, as is the case for most TCRs, but rather are invariant. Some of the antigens that activate iNKT cells are derived from the membrane lipids of microbes and rapidly activate iNKT cells to provide host defense against infection [4]. In addition, antigen presenting cells are stimulated to synthesize endogenous glycolipid antigens in response to the activation of Toll

J. Linden $(\square)$

Division of Inflammation Biology, La Jolla Institute for Allergy and Immunology,

La Jolla, CA, USA

e-mail: jlinden@liai.org like receptors (TLRs) on antigen presenting cells [5]. Although iNKT cells play a protective role in host defense against microbial infections, in diseases such as atherosclerosis, sickle cell anemia and asthma, iNKT cell activation can be deleterious to the host $[6,7]$.

In this issue, Nakou et al. [8] demonstrate that hyperlipidemic individuals have significantly lower numbers of circulating iNKT cells than controls. During 6 months of therapy with $40 \mathrm{mg}$ /day simvastatin, the number of iNKT cells in hyperlipidemic patients increased significantly to near control levels. The increase in circulating iNKT cells in the patients receiving simvastatin appeared not to be due to lipid lowering because there was no relationship between plasma lipids or triglycerides and numbers of iNKT cells in blood. Moreover, the response to another lipid lowering drug, ezetimibe (10 mg/day) was similar to simvastatin in changing plasma lipid profiles of hyperlipidemic patients, but ezetimibe failed to increase numbers of circulating iNKT cells. Unlike statins, which inhibit HMG-CoA, ezetimibe inhibits the absorption of cholesterol in the small intestine. Nakou et al. conclude that the effect of simvastatin to increase circulating iNKT cells in hyperlipidemic patients is not due to the lipid-lowering effects of the statin.

It remains to be determined why simvastatin increases the number of circulating iNKT cells in hyperlipidemic patients and if the increase is of benefit to the patient. Since iNKT cells have been shown to accelerate atherosclerosis progression, the increase in circulating iNKT cells could be viewed as detrimental. However, if simvastatin inhibits the activation of iNKT cells it is likely to reduce the ability of these cells to exacerbate plaque inflammation. Nakou et al. used flow cytometry to measure the expression on iNKT cells of the activation marker, HLA-DR. They found that the percentage of HLA-DR positive iNKT cells in hyperlipidemic patients is significantly higher than in controls, consistent with greater 


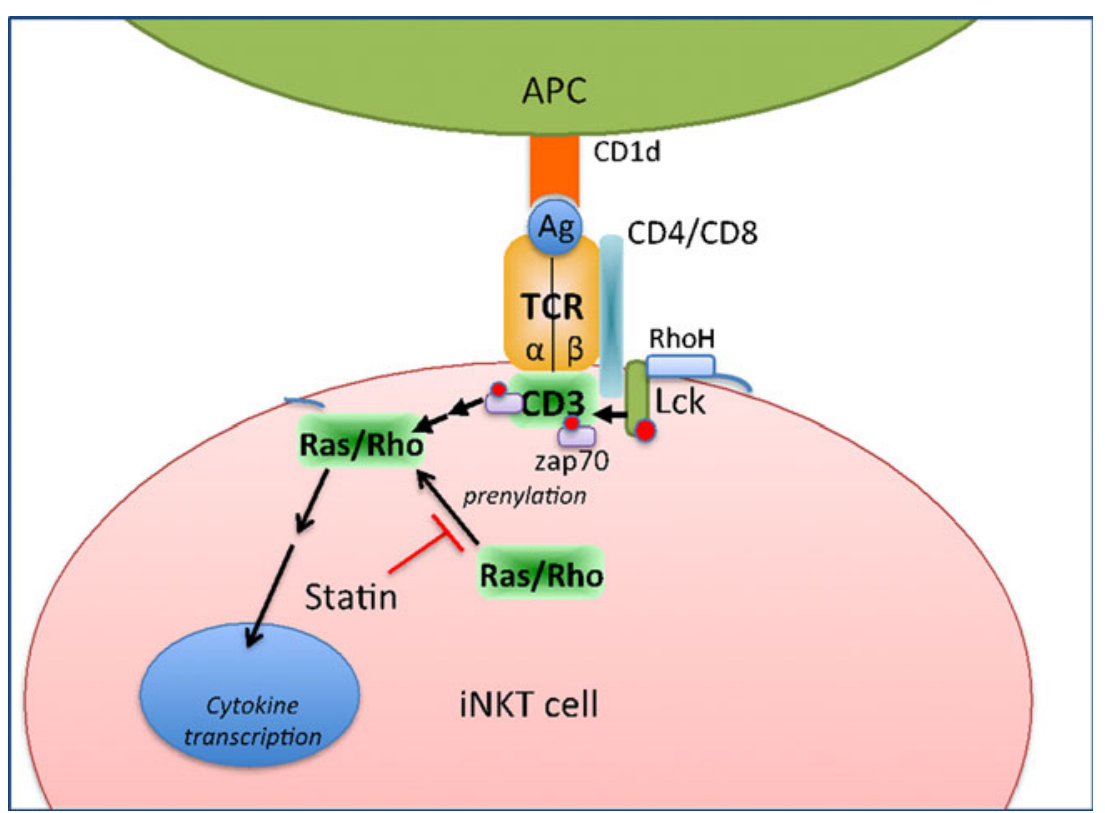

Fig. 1 Effects of statins on iNKT cells. Based on the known effects of statins on $\mathrm{T}$ cells, the study by Nakou et al. suggests that statins may inhibit the activation of iNKT cells and modify cytokine production by reducing the protein prenylation of signaling molecules such as the small G proteins Ras and Rho. Hyperlipidemia results in iNKT cell activation that may occur due to the generation of lipid antigens (Ag) that are presented by CD1d on antigen presenting cells (APCs) to

iNKT activation. Treatment with simvastatin for 6 months lowered the fraction of circulating iNKT cells expressing HLA-DR, but this effect was not statistically significant. Nevertheless, it still remains possible that simvastatin treatment inhibits iNKT cell activation to produce an anti-inflammatory and beneficial effect in patients with atherosclerosis. Nakou et al. point out that by inhibiting iNKT cell activation, statins may increase the number of iNKT cells by reducing activation induced cell death (AICD). It is also possible that statins alter cytokine production by activated iNKT cells. One of the interesting characteristics of iNKT cells is that they can produce both pro-inflammatory Th-1 cytokines such as IFN $\gamma$, and anti-inflammatory Th-2 cytokines such as IL-4. In addition, iNKT cells can inhibit the development of strongly pro-inflammatory Th-17 cells [9] that are thought to play a role in atherosclerosis progression [10,11]. An increase in Th-2 cytokines by iNKT cells could also contribute to another anti-inflammatory and plaque-stabilizing effect of statins, i.e. increased production of regulatory T cells [12]. It is significant also that most iNKT cells in the vascular space are not found in the blood stream but rather are loosely adhered to the walls of blood vessels [13]. It is likely that adhered iNKT cells are more activated than circulating cells. Treatment with simvastatin may alter the fraction of iNKT cells that are found in the circulation, so the number of iNKT cells found in blood is not necessarily indicative of the number of iNKT cells found in inflamed sites such as atherosclerotic plaques. invariant TCRs on iNKT cells. TCR activation initiates a signaling cascade triggered by the activation of lymphocyte-specific protein tyrosine kinase (Lck) and recruitment of Zeta-chain-associated protein kinase 70 (Zap-70) to the immunological synapse that is facilitated by RhoH. Statins, by inhibiting protein prenylation, inhibit the activation of some small $\mathrm{G}$ proteins involved in Zap 70 recruitment and the activation and proliferation of $\mathrm{T}$ cells

In summary, Nakou et al. have demonstrated a novel effect of statins to increase the number of circulating iNKT cells in hyperlipidemic patients. Since iNKT cells can have either a pro-inflammatory or anti-inflammatory character, it remains to be determined if this effect increases or inhibits inflammation in atherosclerotic plaques. However, based on the known effects of statins to inhibit the activation of APCs and lymphocytes, it is likely that the effects of statins on iNKT cells are beneficial for inhibiting the progression of atherosclerosis.

\section{References}

1. Krysiak R, Zmuda W, Okopien B. The effect of ezetimibe, administered alone or in combination with simvastatin, on lymphocyte cytokine release in patients with elevated cholesterol levels. J Intern Med. 2012;271:32-42.

2. Greenwood J, Steinman L, Zamvil SS. Statin therapy and autoimmune disease: from protein prenylation to immunomodulation. Nat Rev Immunol. 2006;6:358-70.

3. Chae HD, Siefring JE, Hildeman DA, Gu Y, Williams DA. RhoH regulates subcellular localization of ZAP-70 and Lck in T cell receptor signaling. PLoS One. 2010;5:e13970.

4. Kronenberg M, Kinjo Y. Innate-like recognition of microbes by invariant natural killer T cells. Curr Opin Immunol. 2009;391-6.

5. Brennan PJ, Tatituri RV, Brigl M, et al. Invariant natural killer $T$ cells recognize lipid self antigen induced by microbial danger signals. Nat Immunol. 2011;12:1202-11. 
6. Field JJ, Nathan DG, Linden J. Targeting iNKT cells for the treatment of sickle cell disease. Clin Immunol. 2011;140:17783.

7. Godfrey DI, Stankovic S, Baxter AG. Raising the NKT cell family. Nat Immunol. 2010;11:197-206.

8. Nakou E, Babageorgakas P, Bouchliou I, et al. Statin-induced immunomodulation alters peripheral invariant natural killer T-cell prevalence in hyperlipidemic patients. Cardiovasc Drugs Ther. 2012;26:this issue.

9. Mars LT, Araujo L, Kerschen P, et al. Invariant NKT cells inhibit development of the Th17 lineage. Proc Natl Acad Sci U S A. 2009;106:6238-43.
10. Chen S, Crother TR, Arditi M. Emerging role of IL-17 in atherosclerosis. J Innate Immunol. 2010;2:325-33.

11. Gao Q, Jiang Y, Ma T, et al. A critical function of Th17 proinflammatory cells in the development of atherosclerotic plaque in mice. J Immunol. 2010;185:5820-7.

12. Mausner-Fainberg K, Luboshits G, Mor A, et al. The effect of HMG-CoA reductase inhibitors on naturally occurring CD4 + CD25+ T cells. Atherosclerosis. 2008;197:829-39.

13. Barletta KE, Cagnina RE, Wallace KL, Ramos SI, Mehrad B, Linden J. Leukocyte compartments in the mouse lung: distinguishing between marginated, interstitial, and alveolar cells in response to injury. J Immunol Methods. 2012;375:100-10. 